\title{
Hysteroscopic resection of retained placental tissue: a feasibility study
}

\author{
T. Dankert • M. Vleugels
}

Received: 16 July 2007 / Accepted: 15 October 2007 / Published online: 14 November 2007

(C) Springer-Verlag 2007

\begin{abstract}
Retained placental tissue (RPT) is a serious cause of secondary postpartum haemorrhage. Various surgical therapies are available with a variety of complications and disadvantages such as uterine perforation, blood transfusions, persisting RPT, recurrence in subsequent pregnancies or Asherman's syndrome. We describe 10 women who underwent hysteroscopic visualisation and resection of RPT. No complications occurred. RPT was histologically confirmed in $90 \%$. No adhesions during a second-look hysteroscopy were seen. All women regained normal menstrual bleedings. Hysteroscopic selective resection of RPT should be considered in patients with secondary postpartum haemorrhage.
\end{abstract}

Keywords Hysteroscopic resection .

Retained placental tissue $\cdot$ Postpartum haemorrhage

\section{Introduction}

Approximately $1-2 \%$ of all singleton deliveries are complicated by retained placental tissue (RPT) [1-3]. Half of all secondary postpartum haemorrhages are caused by retained placental tissue (RPT) [3]. Various drug therapies and surgical therapies to control the bleeding, to clean out the uterus, and to prevent or treat infection are used as treatment. Oxytocics, ergometrine, methotrexate and prosta-

\section{T. Dankert}

Department of Obstetrics and Gynaecology, Radboud University Nijmegen Medical Centre,

Nijmegen, The Netherlands

\section{Vleugels $(\bowtie)$}

Rivierenland Hospital, President Kennedylaan 1, 4002 WP Tiel, The Netherlands

e-mail: vleugels@zrt.nl glandins are used as drug treatment. Surgical interventions to evacuate the uterine cavity include curettage, hysteroscopic loop resection or morcellation [4]. Uterine or hypogastric ligation or embolisation or hysterectomy is used to control the bleeding. Bilateral ligation of the uterine or hypogastric arteries is a useful procedure but complications may occur including the need for significant blood transfusion, persistent retained placental tissue and risk of recurrence in subsequent pregnancies [5]. Since no randomised controlled trials comparing the different surgical treatment options are available, the best management of women with secondary postpartum haemorrhage remains unclear.

Puerperal curettage is complicated by perforation in approximately $3 \%$ of the women $[3,6]$, and placental tissue is only histologically identified in half of patients $[3,6,7]$. Seven percent of the women need further curettage with increased risk of uterine damage $[3,7]$. The hysteroscopic treatment options are mostly described in case reports. In three papers, results of hysteroscopic treatment in more women are described. Tchabo used a hysteroscope to visualise RPT in 95 patients, and no uterine perforation was found. After visualisation, the RPT was removed easily by curettage with polyp forceps. Histology of RPT was confirmed in $100 \%$ of the patients [8]. Cohen et al. compared selective curettage directed by hysteroscopy with conventional blind curettage in women with retained trofoblastic tissue. None of the women who underwent hysteroscopic selective curettage needed a second operation compared with $20.8 \%$ of the women who underwent traditional curettage [9].

In both of these studies the hysteroscopy was still combined with a curettage, but RPT might also be treated with hysteroscopic resection instead of selective curettage directed by hysteroscopy. Goldenberg et al. described 16 patients with retained placental tissue after abortion and 2 
patients after labour [10]. They removed the tissue by using a cutting loop as curette at hysteroscopy. No complications occurred and the bleeding stopped in all cases. Both women who were postpartum underwent a second-look hysteroscopy and intrauterine adhesions were not found. Since these numbers are small, an intensive Pubmed search was done, but no reports or studies with large numbers of hysteroscopic resection of RPT were found. In this report, we present 10 women who underwent a hysteroscopic visualisation and resection of RPT in the past 2 years in the Rivierenland Hospital.

\section{Methods}

In July 2004 hysteroscopic resection of RPT was introduced in our regional public hospital as an alternative treatment option in case RPT was diagnosed.

We retrospectively reviewed the records of women who had a suspicion of RPT and who had a postpartum hysteroscopy between July 2004 and September 2006. Age, obstetric history, delivery details, complaints, ultrasound findings, treatment, and histology were recorded.

The time at which the hysteroscopic resection of RPT was performed ranged from 11 days to 6 months postpartum. The diagnosis of RPT was made by the positive findings on ultrasound scan. The group of women with an early abortion, i.e., before 16 weeks of gestation, has been excluded. Before the 16th week, the treatment policy in these abortions includes conservative treatment with or without Cytotec. Curettage is routine in this group of patients.

Hysteroscopy was conducted with general anaesthesia or spinal anaesthesia using a 9-mm continuous flow resectoscope (Storz equipment, Karl Storz, Tüttlingen, Germany). The hysteroscope was inserted into the uterine cavity under direct video control. Distension of the uterine cavity was created with normal saline. Outflow collection was monitored to avoid fluid overload. After visualisation of the cavity, the location of the retained products was identified and removed with a Versapoint bipolar loop resecting electrode (Gynecare, Johnson \& Johnson, USA). Retained placental tissue was confirmed by the presence of nonvillous trofoblast, chorionic villi or fetal membranes.

Second-look hysteroscopy using a vaginal approach [11] was conducted after 3-6 months at the outpatient department.

\section{Results}

Ten women aged 25-37 years old, five primiparas and five multiparas, were treated by the secondary puerperal pro- cedure (Table 1). The procedure occurred between 11 days and 6 months postpartum. All women had been discharged from the hospital after delivery and readmitted for reasons of bleeding. Four women had had an abortion. None of the multiparas had a history of caesarean section and/or previous postpartum haemorrhage. Two pregnancies were complicated by hypertension (patients D, J). One pregnancy was complicated by premature rupture of membranes (patient B). Six of the women delivered at term $(60 \%)$. The onset of labour was spontaneous in 70\% (7/10) and induced in $30 \%(3 / 10)$. Caesarean section was performed in 2 patients $(20 \%)$. All patients received 5 units of Syntocinon intramuscular in the third stage of labour. Manual extraction of the placenta was performed in $30 \%(3 / 10)$ of the women. No stillbirths occurred. Five $(50 \%)$ patients had primary postpartum haemorrhage.

All women had persistent vaginal bleeding or a secondary postpartum haemorrhage. On request of one patient who had menorrhagia, a conservative policy was chosen. This initial approach explains the time delay in one case.

Ultrasound examination showed intrauterine masses in all patients. Hysteroscopic resection of the RPT was the first treatment in seven patients. One patient was treated first with medroxyprogesterone. A second patient was treated initially with a curettage. The third patient had a curettage twice before the hysteroscopic resection of the RPT was done.

During hysteroscopy, placental tissue was visualised in all patients. In $90 \%(9 / 10)$, the RPT was histologically confirmed, histology of one patient showed only decidual tissue. In none of the histological tissues was myometrial tissue seen. A second-look hysteroscopy 3-6 months later was conducted in 9 of the 10 patients. One patient did not have the second-look hysteroscopy because of a new pregnancy. At hysteroscopy, a normal uterine cavity was seen in all nine patients. All the women had normal menstrual bleedings at control visits. Clinical follow-up was too short to investigate the consequences for subsequent pregnancies and deliveries.

\section{Discussion}

Retained placental tissue is the cause of secondary postpartum haemorrhage in half of patients [3]. Initial treatment consists of oxytocic agents [1]. Various surgical interventions are available if more treatment is required. No randomised controlled trials about different surgical treatments for these patients are available. One of the surgical treatment options for secondary postpartum haemorrhage is curettage. Curettage is very effective but associated with morbidity. Perforation is seen in approximately $3 \%$ of women with postpartum curettage $[3,6]$; severe Asherman's 


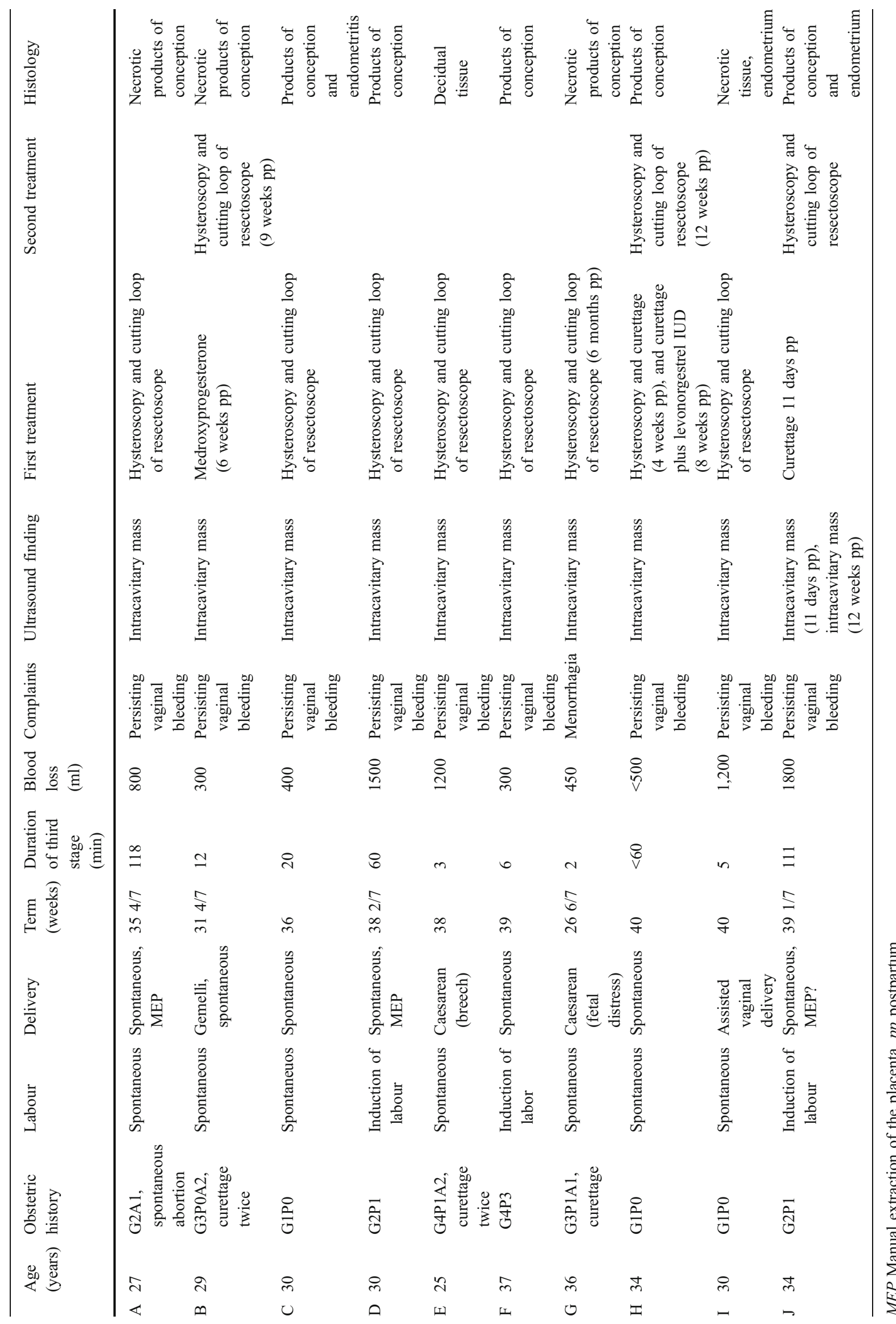


syndrome is seen in $0.5-30 \%$ after secondary curettage for retained placental tissue [7, 12]. Grade II-IV adhesions are associated with infertility, menstrual disturbances, and placenta accretion when pregnancy occurs.

Hysteroscopic treatment options are mostly described in case reports. In the Rivierenland Hospital 10 women underwent hysteroscopic visualisation and resection of RPT because of secondary postpartum haemorrhage in the past 2 years. In all patients the hysteroscopy was without any complications. The RPT was histologically confirmed in $90 \%$ of the patients. All patients had normal menstrual bleedings 3-6 months later. Nine of our patients underwent a second hysteroscopy. The uterine cavity was without adhesions in all cases. Despite the small number of women, this finding might suggest less risk for adhesions after hysteroscopic resection compared to curettage.

Seven percent of women who need a curettage for RPT need a second curettage [3,7]. In a retrospective analysis Cohen described 70 women with residual trophoblastic tissue. All 46 women who underwent a hysteroscopic selective curettage did not need further curettage, but 5 of the $24(20.8 \%)$ women who underwent a traditional curettage required later hysteroscopic operation due to persistent residual tissue. None of our patients needed further treatment after the hysteroscopic resection was performed. The RPT was histologically confirmed in $90 \%$, in comparison with curettage where RPT is confirmed histologically in only half of the women $[3,6,7]$. Incomplete sampling might contribute to underreporting of the incidence of RPT. Tchabo found a $100 \%$ confirmation of histology when the curettage was directed by hysteroscopy.

The number of the women in this article is small, and conclusions from this study should be drawn with caution. In our opinion a hysteroscopic approach should be considered as a treatment option in women with secondary postpartum haemorrhage when RPT is suspected. Either a hysteroscopic selective curettage or a hysteroscopic resec- tion can be done. The advantage of hysteroscopic resection might theoretically be the small risk for adhesions in the uterine cavity. Further research is also needed to determine the consequences for subsequent pregnancies and deliveries.

\section{References}

1. ACOG (1990) Diagnosis and management of postpartum hemorrhage. Int J Gynecol Obstet 36:159-163

2. Dombrowski MP, Bottoms SF, Saleh AA, Hurd WW, Romero R (1995) Third stage of labor: analysis of duration and clinical practice. Am J Obstet Gynaecol 172:1279-1284

3. Hoveyda F, MacKenzie IZ (2001) Secondary postpartum haemorrhage: incidence, morbidity and current management. Br J Obstet Gynaeccol 108:927-930

4. Greenberg JA, Miner JD, O’Horo SK (2006) Uterine artery embolization and hysteroscopic resection to treat retained placenta accreta: a case report. J Minim Invasive Gynecol 13:342-344

5. Verspyck E, Resch B, Sergent F, Marpeau L (2005) Surgical uterine devascularization for placenta accreta: immediate and long-term follow-up. Acta Obstet Gynecol Scand 84:444-447

6. King PA, Duthie SJ, Dong ZG, Ma HK (1989) Secondary postpartum haemorrhage. Aust NZ J Obstet Gynaecol 29(4):394398

7. Pather S, Ford M, Reid R, Sykes P (2005) Postpartum curettage: an audit of 200 cases. Aust NZ J Obstet Gynaecol 45(5):368-371

8. Tchabo JG (1984) Use of contact hysteroscopy in evaluating postpartum bleeding and incomplete abortion. J Reprod Med 29 (10):749-751

9. Cohen SB, Kalter-Ferber A, Weisz BS, Zalel Y, Seidman DS et al (2001) Hysteroscopy may be the method of choice for management of residual trophoblastic tissue. J Am Assoc Gynecol Laparosc 8:199-202

10. Goldenberg M, Schiff E, Achiron R, Lipitz S, Maschiach S (1997) Managing residual trophoblastic tissue: hysteroscopy for directing curettage. J Reprod Med 42:26-28

11. Bettocchi S, Selvaggi L (1997) A vaginoscopic approach to reduce the pain of office hysteroscopy. J Am Assoc Gynecol Laparosc 4 (2):255-258

12. Westendorp IC, Ankum WM, Mol BW, Vonk J (1998) Prevalence of Asherman's syndrome after secondary removal of placental remnants or a repeat curettage for incomplete abortion. Hum Reprod 13(12):3347-3350 